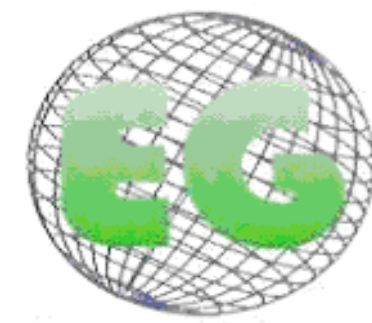

ISSN 1695-6141

$N^{\circ} 23$
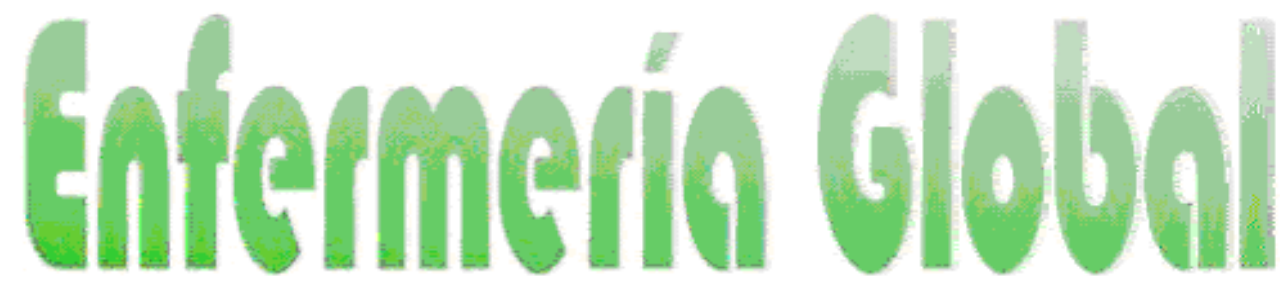

Revista electrónica trimestral de Enfermería

www.um.eslegloball

\title{
La influencia del ritmo cuaternario en la percepción de los estados clínicos y subjetivos de pacientes adultos en hemodiálisis
}

A influência do ritmo quaternário sobre a percepção dos estados clínicos e subjetivos de pacientes adultos em hemodiálise

\author{
"Bechert Caminha, L., **Da Silva, MJP., ***Ribeiro Leão, E. \\ *Graduando do $7^{\circ}$ semestre de Enfermagem. ** Profa. Titular do Departamento de Enfermagem Médico- \\ Cirúrgico da Escola de Enfermagem. Coordenadora do Grupo de Estudos das Práticas Complementares de \\ Saúde do CNPq. *** Doutora en Enfermagem e Pós-Doutorada da Universidade Marc Bloch (França). Vice- \\ coordenadora do Grupo de Estudo das Práticas Complementares de Saúde do CNPq. Universidade de São \\ Paulo. Brasil.
}

Palabras clave: Terapias complementarias; Música; Hemodiálisis; Musicoterapia

Palavras-chave: Terapias complementares; Música; Hemodiálise; Musicoterapia

Keywords: Complementary Therapies; Music; Hemodialysis; Music Therapy.

\section{RESUMEN}

El tratamiento de hemodiálisis requiere de los pacientes intensas restricciones y la necesidad de seguidas sesiones de diálisis que influyen directamente en la calidad de sus vidas. El objetivo de este estudio fue determinar cómo el ritmo cuaternario influye en la percepción del tiempo y las emociones y la aparición de las complicaciones más comunes (hipertensión arterial y dolor) durante la hemodiálisis en relación con la intervención musical. El estudio fue realizado en un hospital privado con 38 pacientes en hemodiálisis, que se sometieron a una sesión de improvisación musical en un teclado, en la que lo subjetivo, el estado clínico y la percepción del tiempo se evaluaron como controles durante la intervención musical y después de la intervención. Más del $80 \%$ de los pacientes se sentían mejor y también notó que el tiempo pasaba más rápido después de la intervención. En el contexto clínico, el estudio sugiere que la música improvisada actúa positivamente, pero no fue un factor decisivo en la diminución de los cuadros de la hipertensión arterial y el dolor.

\section{RESUMO}

O tratamento da hemodiálise impõe aos pacientes intensas restrições e a necessidade das constantes sessões dialíticas influencia diretamente na qualidade de vida dessas pessoas. O objetivo deste estudo foi verificar como o ritmo quaternário influencia a percepção de tempo e emoções, bem 
como a ocorrência das complicações mais comuns (hipertensão arterial e dor) durante uma sessão de hemodiálise em relação à intervenção musical. O estudo foi realizado em um hospital privado, com 38 pacientes em hemodiálise, que se submeteram a uma sessão de improvisação musical em um teclado, nas quais os estados subjetivos, os estados clínicos e a percepção temporal foram avaliados como controle, durante a intervenção musical e após a intervenção. Mais de $80 \%$ dos pacientes se sentiu melhor e também percebeu o tempo passar mais rápido após a intervenção. No âmbito clínico, o estudo sugeriu que a música improvisada agiu positivamente, mas não foi fator decisivo para a diminuição dos quadros de hipertensão arterial e dor.

\section{ABSTRACT}

The treatment of hemodialysis patients requires intense restrictions and the need for constant dialysis sessions directly influences the quality of their lives. The objective of this study was to determine how the pace compounds influences the perception of time and emotions and the appearance of the most common complications (hypertension and pain) during hemodialysis in relation to musical intervention. The study was conducted in a private hospital with 38 patients on hemodialysis, who underwent a session of musical improvisation on a keyboard, in which the subjective, clinical status and time perception were assessed as controls during and after the intervention. More than $80 \%$ of patients felt better and also noticed the time go faster, after the intervention. In the clinical setting, the study suggested that improvised music acted positively, but it was not a decisive factor in the decrease of the tables of hypertension and pain.

\section{INTRODUCCIÓN}

La enfermedad renal crónica es una enfermedad de morbilidad y mortalidad elevada. La incidencia y la prevalencia de la enfermedad en estado terminal está aumentando progresivamente cada año en proporciones epidémicas en Brasil y en el mundo(1).

La insuficiencia renal crónica es una enfermedad definida como la pérdida definitiva de las funciones renales, donde el paciente debe seguir un riguroso tratamiento basado en dietas específicas, control médico y, en general, tratamiento dialítico. El tratamiento impone a los pacientes fuertes restricciones, evidenciando, en consecuencia, diferentes manifestaciones psíquicas y comportamentales, la mayoría de carácter negativo, relacionadas con la máquina de hemodiálisis, que de forma ambigua señala la posibilidad del mantenimiento de la vida social asociada a restricciones, dependencia, frustraciones y temores difusos, incluyendo el temor a la muerte, generando estados estresantes tanto a nivel físico como psíquico (2).

La calidad de vida del paciente renal crónico es muy importante en relación a la supervivencia de estas personas. De entre los diversos factores que comprometen la calidad de vida de esos pacientes, destacamos la realización de sesiones constantes de hemodiálisis. (3)

La hemodiálisis es un método de depuración extracorpórea (realizada en general 3 veces por semana con una duración de 3 a 4 horas cada sesión) por el cual la sangre obtenida por acceso vascular entra en contacto con una solución de diálisis y un filtro especial que usa una membrana semipermeable artificial la cual sustituye a los glomérulos y túbulos renales como filtro. Por medio de esa membrana ocurren intercambios por difusión, filtración y convección. El exceso de agua es quitado de la sangre por ultrafiltración. El circuito extracorpóreo tiene un equipamiento de complejidad variable que prepara, calienta, evalúa y 
proporciona la solución de diálisis, impulsa la sangre con una bomba y monitoriza una serie de parámetros del proceso. Tras la purificación de la sangre, esta es devuelta al cuerpo. (4)

Investigando nuevas formas de cuidado que pudiesen mejorar la asistencia prestada a esos individuos, surgió la utilización de la música como forma de cuidado. La intervención musical proporciona un cuidado más humanizado, además de ser un recurso facilitador de comunicación (5) y tener un nivel elevado de aceptación entre los pacientes (6).

La música forma parte de la naturaleza de los seres humanos, ya que sus componentes básicos como el ritmo, la melodía y la armonía son comunes a la composición de nuestro organismo. Esta semejanza se evidencia al observar el ritmo cardiaco, la sincronización al caminar, la melodía o el volumen de nuestras voces al hablar (7).

La enfermería debe valorar a los pacientes con Insuficiencia renal crónica con tratamiento dialítico como un ser biopsicosocial, ya que los aspectos emocionales son determinantes para el éxito del tratamiento y para la mejora de la calidad de vida (8). La música, según señalan los estudios, intencionadamente bien conducida, puede ser un recurso terapéutico relevante y puede contribuir favorablemente en el individuo ayudándolo a desarrollar nuevas estrategias de enfrentamiento (9) y también puede interferir en los estados subjetivos.

Los estados subjetivos pueden entenderse como hechos de la consciencia caracterizados por diversas percepciones y, en gran parte, por emociones, sentimientos y humor (10). El bienestar subjetivo se refiere a lo que las personas piensan y cómo se sienten con sus vidas, siendo definidos en términos de la experiencia interna del individuo, es decir, es valorado a partir de la perspectiva del propio individuo, a partir de su propio cuadro de referencia (11).

En la gama de estados subjetivos se incluyen, entre otros, la percepción temporal, las emociones y los estados de ánimo, los cuales pueden ser modulados de forma positiva por la música en el ambiente hospitalario (12). El ritmo es el elemento musical que desempeña una función perceptiva importante en el momento presente de toda experiencia musical, ya que se desenvuelve en una matriz temporal. La música está constituida por sonido. El sonido es una onda sonora que se transmite a la atmósfera mediante la forma de una propagación ondulatoria, y nuestro oído es capaz de captarla, haciendo que nuestro cerebro la interprete dándole configuraciones y sentidos. Esa onda sonora es una señal oscilante que recorre y que vuelve por periodos (repitiendo ciertos patrones de tiempo), es decir, obedece a un pulso, razón por la cual se puede pensar en una correspondencia entre las escalas sonoras y las escalas corporales con las que medimos el tiempo (13). Cuando el paciente es sometido a diálisis tiene la percepción de que el tiempo pasa de forma lenta en el periodo de 4 horas, en las cuales la persona permanece "presa" de la máquina dialítica, cuestionándonos si existe algún ritmo específico que fuese percibido de forma más beneficiosa en el enfrentamiento con esa experiencia.

La música tiene una aplicación clínica variada y hay mucha literatura sobre su utilización como recurso terapéutico en la práctica de enfermería. Pero todavía los estudios sobre su uso en la asistencia de los pacientes sometidos a hemodiálisis están bastante restringidos.

Tal escasez de literatura reforzó la necesidad de continuidad del estudio realizado anteriormente, en el que buscamos verificar la influencia del ritmo en la percepción de los estados subjetivos de pacientes adultos sometidos a hemodiálisis. Fueron utilizados 2 ritmos: Marcha y Vals, cuyos compases simples se clasifican en binario y ternario, respectivamente. Los resultados revelaron que el tipo de ritmo parece no haber influido en la respuesta de percepción temporal de los pacientes, ya que ambos llevaron a los pacientes a 
tener sensación de que el tiempo pasó más rápido. Sin embargo, el ritmo influyó en el tipo de experiencia emocional presentada por los pacientes. (14) Otros estudios ya habían demostrado el carácter positivo de esa práctica en cuanto a la alteración de los diferentes estados subjetivos. (15)

Otro aspecto que nos llamó la atención es que tal vez los individuos en el programa de hemodiálisis constituían uno de los grupos de pacientes que tenían su tensión arterial medida el mayor número de veces. Algunos estudios demostraron una prevalencia de hipertensión arterial del $71 \%$ en esa clientela (16). El dolor también es una complicación bastante común (17).

A partir de ahí, nos cuestionamos también el impacto clínico que tales complicaciones pueden traer a los pacientes y si la intervención musical podría ejercer alguna influencia positiva en el enfrentamiento de esas posibles complicaciones (hipertensión y dolor), no investigadas en el estudio anterior.

De esta forma, para profundizar en esa temática en torno a los ritmos, emergió esta investigación sobre el estudio de un ritmo que contenga un compás cuaternario, para que podamos ampliar la discusión sobre esa temática, que favorezca la utilización de la música en el cuidado de los pacientes en programa de hemodiálisis.

\section{OBJETIVOS}

Conocer la influencia del ritmo cuaternario en los estados subjetivos de los pacientes adultos sometidos a hemodiálisis.

Verificar la presencia de complicaciones más comunes durante una sesión dialítica, hipertensión arterial sistémica y dolor, en relación con la intervención musical (la semana antes de la intervención, durante la intervención y la semana después de la intervención).

\section{MÉTODO}

Se trata de un ensayo clínico no controlado realizado en un servicio de hemodiálisis de un hospital general, de tamaño medio de la ciudad de Sao Paulo en diciembre de 2008 y enero de 2009. El proyecto es una continuación del estudio de Iniciación Científica realizado anteriormente sobre esa temática en el mismo servicio de hemodiálisis (14).

La muestra fue constituida por 38 pacientes que presentaron los siguientes criterios de inclusión: adultos sometidos a hemodiálisis, con diagnóstico de insuficiencia renal crónica, sin déficit auditivo referido, sin diagnósticos médicos asociados que implicasen una alteración cognitiva, con comunicación oral preservada y que aceptaran participar en la investigación.

El estudio fue sometido a análisis por el Comité de Ética en Investigación de la Institución y fue aprobado con el número 15/08 y todos los participantes firmaron el Consentimiento Libre y Esclarecido, conforme con la legislación brasileña para la realización de estudios con seres humanos (Resolución Consejo Nacional de Salud 196/96), después de tener conocimiento sobre el objetivo de la investigación. La técnica de "improvisación" fue ejecutada por uno de los investigadores, que es también pianista, con el ritmo propuesto: una Samba-Canción (cuaternario). La escuela eligió la Samba-Canción por tratarse de un género más neutro en relación a los demás géneros que se utilizan de compás cuaternario (rock, bolero, forró, los 
cuales determinan más fuertemente las preferencias musicales, cuando no se excluyen entre sí). Las improvisaciones no fueron realizadas con temas conocidos para minimizar la evocación de memorias personales y así posibilitar una mejor valoración de la influencia del ritmo.

En la Samba-Canción su célula rítmica está formada por cuatro tiempos (1-2-3-4). El pulso es fuerte - debil - fuerte - débil, o sea, el primer tiempo del compás es fuerte, el segundo tiempo es débil, el tercer tiempo es fuerte y el cuarto tiempo es débil .

Las improvisaciones tuvieron una duración de 20 minutos en una única sesión, según el Cuadro 1, compuestos por músicas y sonidos instrumentales ejecutados en un teclado Yamaha PSR 620.

Cuadro 1 - Distribución de los turnos de Hemodiálisis y Ritmo Musical. São Paulo, 2009.

\begin{tabular}{|l|c|c|c|}
\hline Turnos & $1^{\text {a }}$ semana (control) & Semana de intervención & Semana post-intervención \\
\hline $2^{\mathrm{a}}$ mañana & - & Samba-Canción & - \\
\hline $2^{\mathrm{a}}$ tarde & - & Samba-Canción & - \\
\hline $3^{\mathrm{a}}$ mañana & - & Samba-Canción & - \\
\hline $3^{\mathrm{a}}$ tarde & - & Samba-Canción & - \\
\hline
\end{tabular}

Los pacientes fueron divididos en 4 turnos, siendo el primer turno el lunes por la mañana; segundo turno, el lunes por la tarde; tercer turno, martes por la mañana; cuarto turno, martes por la tarde.

Los estados subjetivos de cada paciente fueron valorados la semana de la intervención musical en dos momentos en un mismo día, durante la hemodiálisis, con intervalos de 20 minutos entre las verificaciones, como control. En los días correspondientes a la intervención musical ( $2^{\mathrm{a}}$ semana de intervención), los estados subjetivos fueron valorados inmediatamente antes e inmediatamente después de cada intervención musical, utilizando instrumentos aprobados en estudios sobre el impacto de la música en los estados de ánimo de pacientes hospitalizados (18). La percepción temporal fue valorada por una cuestión específica para que el paciente indicase si el tiempo pasó de forma igual, si fue más rápido o si la sesión de hemodiálisis le pareció que duraba más tiempo.

Fue verificada también la ocurrencia de complicaciones más comunes durante una sesión de hemodiálisis antes y después de la intervención musical, que fueron registradas en el formulario de ocurrencia. Esas complicaciones fueron verificadas una semana antes de las intervenciones, durante las semanas de intervención y una semana después de las intervenciones musicales. Los datos fueron extraídos de la ficha de controles ya existente en el servicio para la monitorizacion clínica.

Los resultados fueron sometidos a análisis estadístico descriptivo y a estadística diferencial. La codificación estadística utilizada fue: X_1_1: antes del control; X_1_2: después del control; X_2_1: antes de la intervención; X_2_2: después de la intervención; X_3_1: antes tras intervencion; X_3_2: después tras intervención. Los tests utilizados fueron McNemarBowker. 


\section{RESULTADOS}

En la muestra de 38 pacientes, la media de edad del grupo estudiado fue de 56 años, con variación de 18 a 85 años. Solo 5 (13\%) tenían menos de 30 años, y 20 (52,6\%) eran mayores (má de 60 años).

En cuanto al género, $57,9 \%$ eran de sexo masculino y $42,1 \%$ de sexo femenino. En relación al tiempo de diálisis al cual los pacientes del estudio estaban sometidos, se constató que 28 pacientes $(73,7 \%)$ presentaban menos de 5 años de diálisis, 6 pacientes $(15,8 \%)$ presentaban entre 5 y 10 años de diálisis y 4 pacientes (10,5\%) más de 10 años. La media de tiempo de participación en el programa de diálisis fue de 3,8 años, con variación de 1 mes a 13,5 años.

En cuanto a la preferencia musical, verificamos una gran diversidad entre los pacientes, variando entre los más diversos estilos musicales. Once de los 38 pacientes (29\%) afirmaron ser eclécticos. Ningún paciente relató que no escuchara música o que no le gustara, lo que demuestra, una vez más, el carácter universal y la gran aceptación de esa práctica complementaria de salud.

La Tabla 1 demuestra y corrobora nuestro estudio anterior ${ }^{(14)}$ que, para la mayoría, la percepción fue de que el tiempo pasó más rápido en relación a la semana anterior (control), con relevancia estadística $(p=0,001)$.

Tabla 1 - Percepción temporal de los pacientes tras sesión musical. São Paulo, 2008/2009.

\begin{tabular}{lcccccc}
\hline Percepción temporal & \multicolumn{2}{c}{ en semanas de estudio } & \multicolumn{2}{c}{ Ritmo Cuaternario } & \multicolumn{2}{c}{ (Samba-Canción) } \\
& \multicolumn{2}{c}{ Control } & \multicolumn{2}{c}{ Semana de Intervención } & \multicolumn{2}{c}{ Semana sin intervención } \\
& $\mathrm{f}$ & $\%$ & $\mathrm{f}$ & $\%$ & $\mathrm{f}$ & $\%$ \\
\hline 1. Más rápido & - & - & 34 & 89,5 & 23 & 60,5 \\
3. Más lento & 3 & 8 & - & - & - & - \\
2. Igual & 35 & 92 & 4 & 10,5 & 15 & 39,5 \\
\hline Total & $\mathbf{3 8}$ & $\mathbf{1 0 0}$ & $\mathbf{3 8}$ & $\mathbf{1 0 0}$ & $\mathbf{3 8}$ & $\mathbf{1 0 0}$ \\
\hline
\end{tabular}

Nuevamente, cuando comparamos con el estudio anterior ${ }^{(14)}$, se observó que la mayoría presentó una mejora en los estados de ánimo en relación a la semana control, según la Tabla 2.

Tabla 2 - Percepción sensorial de bienestar de los pacientes después de la sesión musical. São Paulo, 2008/2009.

\begin{tabular}{lcccccc}
\hline Percepción de bienestar & en semanas de estudio & \multicolumn{2}{c}{ Ritmo Cuaternario } & \multicolumn{2}{c}{ (Samba-Canción) } \\
& \multicolumn{2}{c}{ Control } & \multicolumn{2}{c}{ Semana de } & Intervención & Semana sin intervención \\
& $\mathrm{f}$ & $\%$ & $\mathrm{f}$ & $\%$ & $\mathrm{f}$ & $\%$ \\
\hline 1. Mejor & 2 & 5 & 30 & 79 & 16 & 42 \\
3. Peor & - & - & - & - & - & - \\
2. Igual & 36 & 95 & 8 & 21 & 22 & 58 \\
\hline Total & $\mathbf{3 8}$ & $\mathbf{1 0 0}$ & $\mathbf{3 8}$ & $\mathbf{1 0 0}$ & $\mathbf{3 8}$ & $\mathbf{1 0 0}$ \\
\hline
\end{tabular}


Los principales sentimientos presentados por los pacientes antes y después del ritmo cuaternario propuesto, durante las semanas de estudio, son presentados en la Tabla 3.

Tabla 3 - Estados subjetivos presentes antes y después de la sesión musical. São Paulo, 2008/2009.

\begin{tabular}{lcccccc}
\hline Estados Subjetivos & \multicolumn{2}{c}{ Ritmo Cuaternario } & (Samba-Canción) \\
\hline & \multicolumn{2}{c}{ Control } & & Semana de & intervención & Semana sin intervención \\
\hline Tranquilidad & 15 & 16 & 17 & 17 & 19 & 18 \\
Alegría & 0 & 0 & 3 & 4 & 2 & 2 \\
Calma & 3 & 4 & 5 & 6 & 3 & 4 \\
Paz & 0 & 0 & 0 & 2 & 5 & 5 \\
Distracción & 0 & 0 & 0 & 3 & 0 & 0 \\
Animación & 1 & 1 & 0 & 3 & 1 & 1 \\
Preocupación & 1 & 0 & 2 & 1 & 0 & 0 \\
Tristeza & 9 & 9 & 4 & 1 & 2 & 2 \\
Desanimación & 3 & 3 & 1 & 0 & 1 & 1 \\
Ansiedad & 0 & 0 & 2 & 0 & 1 & 1 \\
Revuelta & 1 & 1 & 1 & 1 & 1 & 1 \\
Rabia & 0 & 0 & 1 & 0 & 0 & 0 \\
Normal & 5 & 4 & 2 & 0 & 3 & 3 \\
\hline Total & $\mathbf{3 8}$ & $\mathbf{3 8}$ & $\mathbf{3 8}$ & $\mathbf{3 8}$ & $\mathbf{3 8}$ & $\mathbf{3 8}$ \\
\hline
\end{tabular}

Se observó que después de la ejecución del ritmo Cuaternario, hubo algunas diferencias en los sentimientos experimentados por los pacientes, aumentando los relatos de sensaciones positivas (tranquilidad, alegría, calma, paz, distracción, animación) y disminuyendo los relatos de sensaciones negativas (preocupación, tristeza, desánimo, ansiedad y rabia). Fue verificada la relevancia estadística entre las semanas control y sin intervención, con $p=0,029$.

En cuanto a la hipertensión arterial, se recogió el valor de la tensión arterial de cada paciente durante la semana anterior a la intervención musical. Considerando los parámetros normales $(120 \times 80 \mathrm{mmHg})$, son presentadas en la Tabla 4 las respectivas clasificaciones de tensión arterial en las semanas de estudio.

Tabla 4 - Clasificación de los pacientes según la tensión arterial y después de la sesión musical. São Paulo, 2008/2009.

\begin{tabular}{lcccccc}
\hline PA & \multicolumn{2}{c}{ Control } & \multicolumn{2}{c}{ Semana de intervención } & Semana sin intervención \\
\hline & antes & después & antes & después & antes & después \\
\hline Normotenso & 10 & 15 & 12 & 20 & 14 & 17 \\
Hipertenso & 24 & 9 & 23 & 4 & 20 & 7 \\
Hipotenso & 4 & 14 & 3 & 14 & 4 & 14 \\
\hline Total & $\mathbf{3 8}$ & $\mathbf{3 8}$ & $\mathbf{3 8}$ & $\mathbf{3 8}$ & $\mathbf{3 8}$ & $\mathbf{3 8}$ \\
\hline
\end{tabular}

Diversos mecanismos pueden estar relacionados con la fisiopatogenia de la hipertensión arterial en la hemodiálisis. Sin embargo, parece haber un consenso de que la etiopatogenia 
de la hipertensión arterial en la hemodiálisis está íntimamente ligada al balance de agua y sodio.

Durante la semana de intervención musical, a la llegada de los pacientes al servicio, la tensión elevada fue algo común. Sin embargo, durante la semana de intervención musical y valoración de la tensión por parte del equipo de enfermería al final de la diálisis, se obtuvo un mayor número de pacientes con su tensión arterial normalizada y un menor número de pacientes saliendo del servicio con un cuadro de tensión arterial elevada, comparados con los de la semana anterior (control). Sin embargo no se observó relevancia estadística $(p=$ 0,375).

Los cuadros algésicos también fueron observados conforme la Tabla 5:

Tabla 5 - Escala de dolor de los pacientes antes y después de la sesión musical . São Paulo, 2008/2009.

\begin{tabular}{lcccccc}
\hline \multirow{2}{*}{$\begin{array}{l}\text { Escala de dolor } \\
\text { intensidad }\end{array}$} & \multicolumn{2}{c}{ Control } & \multicolumn{2}{c}{ Semana de intervención } & Semana sin intervención \\
\cline { 2 - 7 } & antes & después & antes & después & antes & después \\
\hline Ausente (0) & 19 & 18 & 19 & 25 & 21 & 24 \\
Leve (1-3) & 8 & 9 & 7 & 6 & 8 & 5 \\
Moderada (4-7) & 9 & 8 & 10 & 6 & 7 & 7 \\
Intensa (7-10) & 2 & 3 & 2 & 1 & 2 & 2 \\
\hline Total & $\mathbf{3 8}$ & $\mathbf{3 8}$ & $\mathbf{3 8}$ & $\mathbf{3 8}$ & $\mathbf{3 8}$ & $\mathbf{3 8}$ \\
\hline
\end{tabular}

Los cuadros de dolor fueron observados con menor frecuencia durante la semana de intervención musical, aunque no llegaron a presentar relevancia estadística $\left(p=1,000^{\underline{a}}\right)$.

\section{DISCUSIÓN}

La caracterización de la muestra nos permitió observar algunos puntos importantes. El tratamiento dialítico es responsable de un día a día monótono y estricto, y las actividades de los respectivos pacientes se limitan tras el inicio del tratamiento ${ }^{(19)}$. En el lugar de estudio, la televisión y los libros constituyen las únicas formas de entretenimiento de los pacientes, lo que convierte las sesiones diarias extremadamente rutinarias. La inclusión de la música en ese ambiente constituyó otra opción, cuya aceptación fue casi unánime, corroborando la alta aceptación de esa práctica ${ }^{(7)}$.

Conforme la Tabla 2, ningún paciente relató sentirse peor después de la intervención, lo que corrobora la influencia de la música en el bienestar de las personas en el ambiente hospitalario y la gran aceptabilidad de la música en este ambiente ${ }^{18}$.

Cuando comparamos con el estudio anterior sobre esta temática ${ }^{(14)}$, observamos que independientemente del ritmo utilizado (Binario, Ternario o Cuaternario), no hay diferencias de interferencia en la percepción temporal, o sea, todos influyen en esa percepción de la misma forma, pareciendo no haber un ritmo específico que contribuya menos o más. Además, parece que el tipo de ritmo ha sido un factor determinante para el tipo de experiencia emocional que los pacientes presentaron. Tanto el ritmo Binario, como el Ternario y el Cuaternario contribuyeron relevantemente en el aumento del bienestar en detrimento de los sentimientos negativos. Sin embargo, la métrica determinó más fuertemente el énfasis en algunos sentimientos, lo que permite afirmar, por ejemplo, que el 
ritmo Ternario (Vals), de entre el Binario y el Cuaternario, es el más adecuado para proporcionar sensación de paz

En este estudio, llamó la atención la relevancia estadística para la percepción temporal (el tiempo ha pasado más rápido) que se configuró también en la semana subsiguiente a la semana de intervención, lo que no era esperado en relación a la semana control. Eso apunta hacia un efecto carry-over, el cual no sabemos exactamente con quién o con qué puede estar relacionado, ya que puede ser tanto decurrente de la "memoria" de la experiencia vivida anteriormente, o incluso, puede estar influenciado por la "presencia física" del investigador, que estaba todas las mañanas interactuando y tocando al paciente, aunque en esa ocasión no haya desarrollado ninguna intervención fuera de la de valorar al paciente. Tales procesos, de forma aislada o en conjunto, no deben ser descartados y pueden haber sido responsables indirectamente de tales resultados en la última semana de colecta.

La audición musical alegra al oyente y lo devuelve sutilmente ligado al ambiente sonoro. Por eso, la música es, con frecuencia, preferible al silencio, en particular para personas con dolores, porque el silencio (fuera de situaciones meditativas) puede ampliar la consciencia de incomodidad. De entre los mecanismos que se relacionan con ese alivio, encontramos la distracción, la alteración del foco perceptual y la liberación de endorfinas ${ }^{(20)}$, además de la relajación ${ }^{(21)}$. Sin embargo, al contrario de lo que demuestran diversos estudios ${ }^{(12)}$, la audición musical no influyó significativamente en los valores de intensidad dolorosa.

Fuera de la literatura ${ }^{(20)}$ se considera que las ondas cerebrales, frecuencia cardíaca, respiración, tono emocional y otros ritmos orgánicos pueden cambiar sutilmente según lo que se oye ${ }^{(20)}$, en este estudio no se observó influencia significativa de la música en la experiencia de dolor ni en la tensión arterial. Los resultados sugieren que la música actuó positivamente en todos los ámbitos estudiados, pero no fue factor primordial para que los cuadros fisiológicos (Tensión Arterial y Dolor) estuviesen alterados hacia valores más próximos de los deseados.

\section{CONSIDERACIONES FINALES}

Este estudio permitió verificar y confirmar algunas propuestas que ya habían sido observadas en un estudio anterior sobre la misma temática ${ }^{(14)}$. Independientemente del ritmo utilizado, este es capaz de alterar la percepción temporal de las personas e influir significativamente en la sensación de tiempo que cada uno lleva consigo.

Por otro lado, dependiendo del tipo de ritmo escuchado, podemos tener diferentes experiencias emocionales. A pesar de esas diferencias de sentimientos experimentados, el ritmo cuaternario se mostró adecuado para provocar sensaciones positivas en detrimento de sentimientos negativos.

Tras la ejecución, no hubo mayor normalización de la tensión arterial o de sensible mejoría en los cuadros de dolor relatados por los pacientes.

Para concluir, a continuación relatamos aspectos que no fueron objetivados en ese estudio, pero que fueron significativos para el autor que desarrolló la intervención musical durante la realización de la investigación y por considerar también que puedan suscitar nuevos objetivos de investigación.

Una vez más, como en el último estudio de $2007^{(14)}$ desarrollado en el mismo servicio de Hemodiálisis, la aceptación por parte de los pacientes fue asombrosa. Pocos pacientes que 
participaron del estudio anterior participaron nuevamente en esta investigación. Algunos cambiaron de horarios, otros ya realizaron el trasplante, pero la gran mayoría se mudó a otras instituciones de salud para continuar con el tratamiento dialítico. Durante la intervención musical totalmente improvisada dentro de las escalas que el investigador decidió utilizar, muchos cantaban frases, como si la música existiera ya y no fuese fruto de la creatividad del investigador que estaba allí ejecutando música en vivo.

Un aspecto interesante para ser observado es que los beneficios que la música trae se acentúan con la presentación en vivo. La presencia del investigador que estuvo todas las mañanas y tardes durante Diciembre de 2008 y Enero de 2009 (épocas consideradas festivas, donde englobamos Navidad y Año Nuevo) parece haber funcionado como estrategia de acogida para los pacientes, disminuyendo sentimientos como la soledad que es bastante común durante las sesiones dialíticas. La interacción humana forma parte y es una variable que, aparte de su dificultad para ser medida, está presente en la investigación desarrollada en el ambiente clínico.

No pasó mucho tiempo para que los pacientes, al final de la intervención musical, se aproximaran más, pidieran músicas más específicas de los más diversos estilos, intentaban tocar algunas notas y hasta se interesaran por clases de música particulares. La reciprocidad por parte de los pacientes era notoria y muchos de ellos no creían que la intervención musical con duración inaplazable de 20 minutos "durara 20 minutos". Muchos llegaron a desconfiar, diciendo que la ejecución no había durado ni 5 minutos. Eso nos resume claramente la sensación referida de que el tiempo "pasó más rápido".

De la misma forma que en el estudio pasado, hubo pacientes que lloraron durante y al terminar la intervención musical, reforzando nuestra observación anterior, de que aun improvisando melodías diferenciadas y desconocidas, ellos eran capaces de tener los más diversos estímulos de la imaginación y evocar recuerdos pasados.

Uno de los pacientes era músico. Tal vez por su audición más afinada y diferenciada de los demás, sus expresiones no verbales eran especiales. Él cerraba los ojos y, nítidamente, iba para dentro de su propio mundo, su propio yo, sin hablar nada, simplemente oyendo. Él volvía a interactuar cuando la intervención musical paraba. Por curiosidad, percibiendo sus expresiones no verbales, durante el improvisado dentro de una escala menor armónica, ocurrió un "error" a propósito en una secuencia específica, desafinando dentro de una escala musical que estaba siendo explorada. En ese momento, él abrió los ojos espantado, miró fijamente para el ejecutante sin entender, y ambos empezaron a reír. La necesidad de unir la teoría musical dentro de la práctica es esencial para que la música tenga sentido y posibilite interpretaciones propias. Notas desafinadas e improvisación sin sentido no tienen ningún efecto, por el contrario, provocan espanto y agitación, como si los oidos "doliesen", lo que puede interferir en ese "viaje" dentro del propio yo. Un asunto como ese merece ser investigado oportunamente con juicioso rigor metodológico.

El estudio, igual que el estudio anterior sobre este tema, presenta las mismas limitaciones: la imposibilidad de aislar en la intervención realizada, solamente el ritmo de los demás elementos sonoro-musicales inherentes, por la utilización de un instrumento armónico. Es probable que estudios diseñados únicamente con instrumentos de percusión puedan revelar mayor exactitud para ese tipo de análisis.

A partir de los resultados obtenidos, consideramos la música como un potencial que debería ser más perfeccionado y explorado, atendiendo a una mejor calidad de vida y una mayor 
cualificación del cuidado dentro del ambiente, percibido muchas veces como "triste" y con procedimientos que parecen "durar mucho tiempo" en la Hemodiálisis.

\section{REFERENCIAS}

1. Sesso R. Epidemiologia da Doença Renal Crônica no Brasil e sua prevenção. 2006; Disponível em: <http://www.cve.saude.sp.gov.br/htm/cronicas/irc prof.htm>. Acesso em 19 jan 2009.

2. Angerami VA. Novos rumos na Psicologia da saúde. ed. Pioneira Thompson Learning. São Paulo, 2002.

3. Castro M, Caiuby AVS, Draibe SA, Canziani MEF. Qualidade de vida de pacientes com insuficiência renal crônica em hemodiálise avaliada através do instrumento genérico SF-36. Rev. Assoc. Med. Bras. 2003; 49(3): 245-249.

4. Smeltzer SC, Bare BG. Brunner \& Suddarth: tratado de enfermagem médico-cirúrgica. 10 ed. Rio de Janeiro: Guanabara Koogan; 2006.

5. Pinheiro MCD, Brito MF, Jorge JS, Lopes MM, Rodrigues FAC. Música e saúde mental: relato de experiência de acadêmicos de enfermagem. Rev. Técnico Científica Enfermagem 2003; 1(1):14-7.

6. Mccaffery M. Nursing approaches to nonpharmacologial pain control. Int J Nurs Stud. 1990; 27(1): 1-5.

7. Backes DS. et al. Música: terapia complementar no processo de humanização de uma CTI. Rev. Nursing. 2003; 66(6): 37-42.

8. Canhada, S.J.G. Aspectos emocionais dos pacientes em hemodiálise crônica. 1990. Monografia (Graduação) - Universidade Federal de Pelotas/FEO, Pelotas, 1990.

9. Poslusny SM. Street music or the blues? The lived experience and social environment of depression. Public Health Nurs 2000; 17(4): 292-9.

10. Engelmann, A. Da conceituação de estado subjetivo até a proposição dos escalões de percepto. Psicologia: Reflexão e Critica. 2002; 15: 393-405.

11. Diener E, Suh E, Oishi S. Recent findings on subjective well-being. Indian Journal of Clinical Psychology. 1997; 24: 25-41.

12. Leão ER, Silva MJP. A música como intervenção de enfermagem no controle da dor. In: Chaves LD, Leão ER. Dor $5^{\circ}$ sinal vital: reflexões e intervenções de enfermagem. 2 ed. São Paulo: Martinari; 2007.

13. Wisnik JM. O som e o sentido: outra história das músicas. São Paulo: Companhia das Letras; 1999. 
14. Caminha LB, Silva MJP, Leão ER. A influência de ritmos musicais sobre a percepção dos estados subjetivos de pacientes adultos em Hemodiálise. Rev Esc Enf USP. v.43, n.4. No prelo 2009.

15. Silva SA, Fava SMCL, Nascimento MS, Ferreira CS, Marques NR, Alves SM. Efeito terapêutico da música em portador de Insuficiência Renal Crônica em Hemodiálise. Rev. Enferm. UERJ, Rio de Janeiro. 2008; 16(3): 382-7.

16. Salem MM. Hypertension in the hemodialysis population: A survey of 649 patients. Am J Kidney Dis 26: 461-468, 1995.

17. Bastos MCM. Atualização em hemodiálise: complicações agudas em Hemodiálise.Jornal Brasileiro de Nefrologia 2001; 23(2): 108-13.

18. Leão ER, Aquino CR, Bussoti EA, Ribeiro EFF. Uma canção no cuidar: a música humanizando a assistência. Anais eletrônico 9. Enftec; 2004.

19. Martins MRI, Cesarino CB. Qualidade de vida de pessoas com doença renal crônica em tratamento hemodialítico. Rev. Latino Am Enfermagem 2005; 13: 670-6.

20. Campbell D. O efeito Mozart. Rio de Janeiro: Rocco; 2001.

21. Paula AAD, Carvalho EC, Santos CB. The use of the progressive muscle relaxation technique for pain relief in gynecology and obstetrics. Rev Latino-am Enfermagem 2002; 10(5):654-9.

ISSN 1695-6141 